Association for Information Systems AIS Electronic Library (AISeL)

Wirtschaftsinformatik Proceedings 1999

Wirtschaftsinformatik

February 1999

\title{
Die Bewertung von IV-Entwicklungsprojekten mit Methoden der Optionspreistheorie
}

Eberhard Stickel

Universität Frankfurt/Oder, stickel@euv-frankfurt-o.de

Follow this and additional works at: http://aisel.aisnet.org/wi1999

\section{Recommended Citation}

Stickel, Eberhard, "Die Bewertung von IV-Entwicklungsprojekten mit Methoden der Optionspreistheorie" (1999).

Wirtschaftsinformatik Proceedings 1999. 36.

http://aisel.aisnet.org/wi1999/36

This material is brought to you by the Wirtschaftsinformatik at AIS Electronic Library (AISeL). It has been accepted for inclusion in Wirtschaftsinformatik Proceedings 1999 by an authorized administrator of AIS Electronic Library (AISeL). For more information, please contact elibrary@aisnet.org. 


\section{Die Bewertung von IV-Entwicklungsprojekten mit Methoden der Optionspreistheorie}

Eberhard Stickel

Universität Frankfurt/Oder (stickel@euv-frankfurt-o.de)

\section{Inhalt}

1 Einleitung

2 Probleme beim Einsatz der Kapitalwertmethode

3 Beurteilung von Pilotprojekten mit der Optionspreistheorie

4 Beurteilung von mehrstufigen Entwicklungsprojekten

5 Simulation

6 Ausblick

7 Zusammenfassung 


\begin{abstract}
Die Beurteilung der Wirtschaftlichkeit von IV-Entwicklungsprojekten ist ein bedeutsames aber leider bisher nur rudimentär gelöstes Problem. Schwierigkeiten bereitet unter anderem die Monetarisierung von Kosten- und Nutzeffekten sowie die daran anschließende Entscheidungsaufbereitung. Mit klassischen Methoden der Investitionsrechnung können im allgemeinen Handlungsspielräume eines Entscheidungsträgers nicht bewertet werden. Derartige Handlungsspielräume sind aber insbesondere bei gut gegliederten IV-Entwicklungsprojekten in nennenswertem Umfang vorhanden. Beispiele sind die Möglichkeit des Abbruchs am Ende einer Phase oder die Möglichkeit der Kapazitätsausweitung nach Durchführung eines Pilotprojektes. Derartige Handlungsspielräume können den Wert eines Projektes nachhaltig erhöhen, beziehungsweise ein derartiges Projekt erst rechtfertigen. Zur Bewertung von Handlungsspielräumen kann auf Ansätze der Optionspreistheorie zurückgegriffen werden. Die klare Struktur von IV-Entwicklungsprojekten erlaubt dabei eine leichtere Umsetzung der Theorie in die Praxis. Dies wird im vorliegenden Beitrag näher ausgeführt.
\end{abstract}

\title{
1 Einleitung
}

Die Beurteilung der Wirtschaftlichkeit von IV-Entwicklungsprojekten ist ein in der Praxis äußerst bedeutsames Problem. Dabei sind einerseits der Entwicklungsaufwand und andererseits die mit dem fertigen Produkt verbundenen Nutzeffekte zu schätzen. Schätzen bedeutet in diesem Zusammenhang im allgemeinen die Angabe monetärer Größen. Erst dann sind bekannte Methoden der Investitionsrechnung anwendbar. Bereits die Projektaufwandschätzung ist ein schwieriges Unterfangen. In besonderem Maße gilt dies für die Beurteilung und Monetarisierung der Nutzeffekte. Die methodische Unterstützung für Wirtschaftlichkeitsanalysen von Entwicklungsprojekten ist unzureichend (vgl. etwa Schumann 1993). So existieren zahlreiche einzelne Methoden zur Ermittlung einzelner Effekte (etwa das hedonistische Modell zur Beurteilung der monetären Wirkungen von Tätigkeitsverschiebungen; vgl. dazu Stickel 1992), eine Integration dieser Teilmodell ist bisher allerdings nicht oder nur unzureichend gelungen.

Gelegentlich wird in der Literatur der Sinn von Wirtschaftlichkeitsanalysen auf der Basis monetärer Größen gänzlich angezweifelt (siehe dazu etwa die Ausführungen in Antweiler 1995). Vorgeschlagen wird dann häufig der Einsatz multikriterieller Verfahren. Eine bekannte Ausprägung derartiger Verfahren ist die Nutzwertanalyse (vgl. Zangemeister 1976). Anzumerken ist allerdings, daß zur Entscheidungsfindung letztendlich alle Kriterien zusammengefaßt werden müssen. Bei multikriteriellen Verfahren ist nahezu immer die willkürliche 
Gewichtung der Zielbeiträge zu kritisieren. Verfahren, die eine Bewertung auf Basis monetärer Größen vornehmen, weisen hingegen dieses Defizit nicht beziehungsweise nicht in gleichem Umfang auf. Schließlich sei auch darauf hingewiesen, daß im Rahmen der wertorientierten Unternehmensführung die Beurteilung von Entwicklungsprojekten auf der Basis monetärer Größen unerläßlich ist (vgl. Copeland et al. 1996).

Im folgenden soll unterstellt werden, daß eine monetäre Bewertung von Kostenund Nutzeffekten eines Entwicklungsvorhabens möglich und gewollt ist. Sind die Zahlungsströme geschätzt, kann auf die klassischen Methoden der Investitionsrechnung zurückgegriffen werden. Dabei erfüllt nur die Kapitalwertmethode die nachfolgend aufgeführten Mindestanforderungen an ein Investitionsrechenverfahren (vgl. Copeland/Weston 1992, S. 25 ff.):

1. Berücksichtigung aller Zahlungsströme. Dies bedeutet, daß alle während des Planungshorizonts anfallenden Zahlungen durch das Verfahren berücksichtigt werden. Gegen diese Forderung verstößt beispielsweise das Amortisationsdauerverfahren, wo Zahlungen nach Amortisation nicht berücksichtigt werden.

2. Berücksichtigung der Zeitstruktur der Zahlungen. Dies erfordert die Verwendung von Diskontierungszinssätzen, um Zahlungen, die zu unterschiedlichen Zeitpunkten anfallen, vergleichbar zu machen. Statische Verfahren der Investitionsrechnung verletzen dieses Kriterium.

3. Wertadditivitätsprinzip. Separate Entwicklungsprojekte können separat oder zusammen (durch Addition der Zahlungsreihen) bewertet werden. Der Wert des zusammengesetzten Projektes soll sich dann als Summe der Werte der Einzelprojekte ergeben. Insofern sind Synergien zwischen Projekten ausgeschlossen. Dies stellt allerdings eine Grundannahme der klassischen Investitionsrechnung dar. Ein Projekt muß vollkommen separierbar sein. Alle Kosten- und Nutzeffekte müssen eindeutig dem Projekt zuzurechnen sein. Gegen das Prinzip der Wertadditivität verstößt unter anderem die interne Zinsfußmethode.

Mit dem praktischen Einsatz der Kapitalwertmethode sind einige Probleme verbunden. Schwierigkeiten bereitet zum einen die Bestimmung geeigneter risikoangepaßter Diskontierungsfaktoren. Zum anderen erlaubt es die Kapitalwertmethode nicht, Handlungsspielräume zu bewerten. Gerade bei IVEntwicklungsprojekten liegen zahlreiche Handlungsspielräume vor. Wegen der üblicherweise guten Strukturierung des Entwicklungsprozesses in einzelne Entwicklungsphasen (Vorgehensmodelle) kann am Ende einer jeden Phase über die Weiterführung des Projektes auf Basis aktualisierter Daten entschieden werden. Es besteht also die Möglichkeit des Abbruchs beziehungsweise der Weiterführung. Insofern liegen hier wertsteigernde Abbruch- beziehungsweise Weiterführungsoptionen vor. Darüber hinaus erlaubt die gute Strukturierung eine relativ einfache Bewertung der Handlungsspielräume (bei weniger gut strukturierten Projekten ist die Bewertung im allgemeinen mathematisch 
wesentlich aufwendiger). Insofern ist die hier präsentierte Theorie der Realoptionen für den Einsatz im Rahmen der IV-Wirtschaftlichkeitsanalyse geradezu prädestiniert.

Um Erfahrungen mit neuen Entwurfsparadigmen und/oder neuen IVTechnologien zu sammeln erfreuen sich Pilotprojekte einer großen Beliebtheit. Der Kapitalwert eines Pilotprojektes ist in nahezu allen Fällen negativ (vgl. Kambil et al. 1995). Erst die Möglichkeit, nach Durchführung des Pilotprojektes über die Weiterführung (im allgemeinen verbunden mit einer Kapazitätsausweitung) entscheiden zu können, schafft einen positiven Projektwert. Dieser Handlungsspielraum kann, dies wird in einem der folgenden Abschnitte gezeigt, als Kapazitätsausweitungsoption in Verbindung mit einer Abbruchoption, interpretiert werden.

Im vorliegenden Beitrag wird gezeigt, wie das Instrumentarium der Kapitalwertmethode sinnvoll um Ansätze der Optionspreistheorie ergänzt werden kann. Es ergeben sich dabei realistischere Projektwerte. Diese Projektwerte liegen im allgemeinen deutlich über den Projektwerten, die mittels der Kapitalwertmethode geschätzt werden. Der Einsatz der Optionspreistheorie kann natürlich das nach wie vor bestehende Schätzproblem in bezug auf die benötigte Zahlungsreihe nicht lösen. Allerdings können die zugrunde liegenden Annahmen über Parameterwerte durch leicht implementierbare Simulationsverfahren modifiziert werden. Im Rahmen derartiger Sensitivitätsanalysen erhält man dann im allgemeinen belastbares Zahlenmaterial, welches innerhalb gewisser Bandbreiten sinnvolle Aussagen über die Wirtschaftlichkeit von Entwicklungsprojekten ermöglicht.

Im folgenden zweiten Abschnitt wird auf die Probleme des Einsatzes der Kapitalwertmethode nochmals detaillierter eingegangen. Daran anschließend wird im Rahmen eines ersten Anwendungsfalles der Einsatz der Optionspreistheorie zur Bewertung von Pilotprojekten diskutiert. Darauf aufbauend erfolgt im vierten Teil die Betrachtung eines in vier Phasen gegliederten Entwicklungsprojektes. Der fünfte Abschnitt beschäftigt sich mit Sensitivitätsanalysen und der Notwendigkeit einer angemessenen IV-Unterstützung beim Einsatz der Optionspreistechniken. Der sechste Abschnitt enthält einen Ausblick. Schließlich erfolgt im siebten Abschnitt eine Zusammenfassung der präsentierten Ergebnisse.

\section{Probleme beim Einsatz der Kapitalwertmethode}

Für das Entwicklungsprojekt sollen Zahlungsströme in Form einer Zahlungsreihe vorliegen. Eine derartige Zahlungsreihe kann als Vektor

$Z=\left(z_{0}, z_{1}, \ldots, z_{T}\right)$

interpretiert werden. Dabei bezeichnet $T$ den Planungshorizont, die Größen $z_{i}$ sind die saldierten Ein- beziehungsweise Auszahlungen der $i$-ten Periode. 
Zur Berechnung des Kapitalwertes benötigt man einen Abzinsungsfaktor $r$. Der Kapitalwert der obigen Zahlungsreihe ist dann gegeben durch

$$
K=\sum_{t=0}^{T} z_{t}(1+r)^{-t} .
$$

Zahlungen zum Zeitpunkt $t$ werden folglich $t$ Perioden mit dem Zinssatz $r$ abgezinst. Die Bestimmung des Zinssatzes $r$ ist ein in der Literatur intensiv diskutiertes Problem. So soll $r$ zum einen die Kapitalkosten reflektieren. Andererseits soll $r$ auch dem individuellen Projektrisiko Rechnung tragen. Im allgemeinen kann man ja nicht unterstellen, daß es sich bei den Zahlungen in $Z$ um sichere Größen handelt. Vielmehr ist davon auszugehen, daß die Größen $z_{t}$ stochastisch sind. Die moderne Finanzierungstheorie stellt mit dem Capital Asset Pricing Modell einen theoretischen Rahmen zur Bewertung risikobehafteter Zahlungen zur Verfügung (vgl. Spremann 1996, S. 533 ff.). Der erwartete Ertrag $\mu$ eines Projektes ist dabei eine lineare Funktion des nicht diversifizierbaren Projektrisikos. Präziser gilt

$$
\mu=r_{f}+\left(\mu_{M}-r_{f}\right) \frac{\rho \sigma}{\sigma_{M}} .
$$

Dabei sind $r_{f}$ der risikofreie Zinssatz, $\mu_{M}$ und $\sigma_{M}$ die Rendite beziehungsweise Standardabweichung des Marktportfolios (gemäß der Portfoliotheorie), welches aus allen risikobehafteten Anlagen besteht, $\sigma$ die Standardabweichung der Projektrückflüsse und $\rho$ die Korrelation der Projektrückflüsse mit den Erträgen des Marktportfolios. Die so errechnete Rendite $\mu$ ist nach der Theorie der korrekte risikoangepaßte Diskontierungsfaktor. $\mathrm{Zu}$ beachten ist allerdings, daß das CAPM ein Einperiodenmodell darstellt. Eine Erweiterung auf mehrere Perioden ist nicht beziehungsweise nur unter großen Schwierigkeiten möglich. Beispielsweise muß man davon ausgehen, daß die Projektrückflüsse einzelner Perioden miteinander korreliert, also nicht stochastisch unabhängig sind (Pfadabhängigkeit). Deshalb sind für jede Periode und für jeden potentiell möglichen Umweltzustand spezifische risikoangepaßte Diskontierungsfaktoren zu bestimmen (vgl. Copeland/Weston 1992 S. 401 ff.). Dies stellt für die Praxis ein sicherlich nicht lösbares Problem dar. Aus diesem Grunde werden häufig Mischzinssätze, welche die unterschiedlichen Kosten des Eigen- beziehungsweise Fremdkapitals reflektieren und einen Risikozuschlag enthalten, verwendet (vgl. Kilka 1995, S. 11 ff.).

Die Optionspreistheorie versucht, diese Problematik durch den Ansatz der arbitragefreien Bewertung zu umgehen. Eine Option stellt ein Recht aber keine Verpflichtung dar, einen der Option zugrunde liegenden Gegenstand (Finanzoder Warentitel, im Falle von Realoptionen aber auch Investitionsprojekte) innerhalb eines festgelegten Zeitraumes (amerikanische Option) oder zu einem bestimmten Zeitpunkt (europäische Option) zu einem bestimmten Basispreis zu 
kaufen (Calloption) beziehungsweise zu verkaufen (Putoption). Einen detaillierten Überblick bietet Hull (vgl. Hull 1998).

Grundgedanke der arbitragefreien Bewertung ist die Überlegung, daß risikolose Erträge, die über die Verzinsung $r_{f}$ hinausgehen, unmöglich sind (vgl. Spremann 1996, S. 557 ff.). Andernfalls setzt ein Arbitrageprozeß ein, der für die Wiederherstellung dieses Zusammenhangs sorgt. Dies stellt einerseits einen wesentlichen Vorteil der Optionspreistheorie dar. Andererseits ist aber genau diese Prämisse der größte Nachteil bei der Übertragung der Optionspreistheorie auf realwirtschaftliche Problemstellungen. Vorausgesetzt werden muß nämlich die Existenz eines Marktpreises für das zugrunde liegende Investitionsprojekt (vgl. Laux 1993). Im Bereich der Finanzwirtschaft sind die den einzelnen Optionen zugrunde liegenden Finanztitel handelbar. Damit existieren Marktpreise. Es ist nicht auf den ersten Blick einsichtig, was diese Restriktion für die Übertragung des Optionspreisansatzes auf Investitionsprojekte, also auf den leistungswirtschaftlichen Bereich eines Unternehmens, bedeutet. Darauf wird im nächsten Abschnitt zurückzukommen sein.

\section{Beurteilung von Pilotprojekten mit der Options- preistheorie}

Ein Unternehmen kann im Rahmen eines Pilotprojektes Erfahrungen im Umgang mit einem neuen Entwicklungsparadigma beziehungsweise einer neuen Entwicklungstechnologie sammeln. Dazu ist eine Anfangsinvestition in Höhe von $S_{1}$ Geldeinheiten erforderlich. Nach Ablauf einer Zeitperiode kann das begonnene Projekt unter Aufstockung der Kapazitäten weitergeführt, aufgeschoben oder abgebrochen werden. Im folgenden wird der Fall betrachtet, daß nach einer Periode die Entscheidung über Abbruch oder Weiterführung zu fällen ist. Im Falle der Weiterführung ist eine weitere Investitionsrate in Höhe von $S_{2}$ Geldeinheiten zu erbringen. Zu diesem Zeitpunkt ist allerdings bekannt, ob sich das neue Entwurfsparadigma beziehungsweise die neue Technologie "durchsetzen" konnte. Ist dies der Fall, rechnet das Unternehmen mit künftigen Rückflüssen von $R_{u}$ Geldeinheiten (günstiger Fall), im ungünstigen Fall mit $R_{d}$ Geldeinheiten. Aus heutiger Sicht sollen beide Möglichkeiten gleich wahrscheinlich sein.

Zunächst wird die Kapitalwertmethode angewendet. Die erwarteten Rückflüsse ergeben sich durch Bildung des arithmetischen Mittels (Bildung des Erwartungswertes). Der zum (vollständigen) Projekt gehörende Zahlungsstrom ist dann gegeben durch

$$
Z=\left(-S_{1},-S_{2}+\frac{1}{2}\left(R_{u}+R_{d}\right)\right) .
$$


Der Kapitalwert ergibt sich bei Verwendung eines Diskontierungssatzes $r \mathrm{zu}$

$$
K=-S_{1}-S_{2}(1+r)^{-1}+\frac{1}{2}\left(R_{u}+R_{d}\right)(1+r)^{-1} \text {. }
$$

Zur einfacheren Analyse soll im folgenden $S_{1}=5.000, S_{2}=30.000, R_{u}=45.000$ und $R_{d}=21.000$ unterstellt werden. Darüber hinaus wird $r=0,05$ angenommen. Mit diesen Zahlenwerten ergibt sich der Kapitalwert $K=-2.142,86$. Folglich lohnt die Durchführung des Projektes nicht. Eine sinnvollere Vorgehensweise wäre allerdings sicherlich das Erbringen der Anfangsinvestition $S_{1}$. Je nach eintretendem Umweltzustand wird dann das Projekt mit erhöhter Kapazität weitergeführt und es ergeben sich Rückflüsse in Höhe von $R_{u}$ oder das Projekt wird eingestellt. Im letzten Fall ergeben sich keine Rückflüsse, allerdings ist in diesem Fall auch die zweite Investitionsrate $S_{2}$ nicht zu erbringen. Aufgrund der einfachen Struktur des gewählten Beispiels kann man den "neuen" Projektwert leicht errechnen. Er ist gegeben durch

$$
K^{*}=-S_{1}+\frac{1}{2}\left(-S_{2}+R_{u}\right)(1+r)^{-1}=-5000+\frac{7500}{1+r} \text {. }
$$

Dieser Wert ist positiv, solange $r<0,5$ gilt.

Die eben skizzierte Vorgehensweise entspricht im wesentlichen einer Entscheidungsbaumanalyse (Bamberg/Coenenberg 1996, S. 232 f.). Wichtig für die Anwendbarkeit dieses Verfahrens ist die Kenntnis beziehungsweise Schätzung der Eintrittswahrscheinlichkeiten der unsicheren Umweltzustände (im Beispiel wurde von einer Wahrscheinlichkeit von 0,5 für das Eintreten des positiven beziehungsweise negativen Umweltzustandes ausgegangen). Ebenso muß der Diskontierungszinssatz $r$ festgelegt werden. Das Investitionsvorhaben kann man nun allerdings auch als Option interpretieren. Gegen Zahlung von $S_{1}$ erwirbt das Unternehmen eine Calloption auf das Investitionsprojekt zum Basispreis $S_{2}$. Gegen Zahlung von $S_{2}$ kann folglich zum Zeitpunkt $t=1$ das Projekt in größerem Umfange fortgesetzt werden. Die Option kann allerdings auch verfallen, falls eine Fortführung zum Zeitpunkt $t=1$ nicht sinnvoll ist. In diesem Fall ist die bezahlte Optionsprämie in Höhe von $S_{1}$ verloren. 


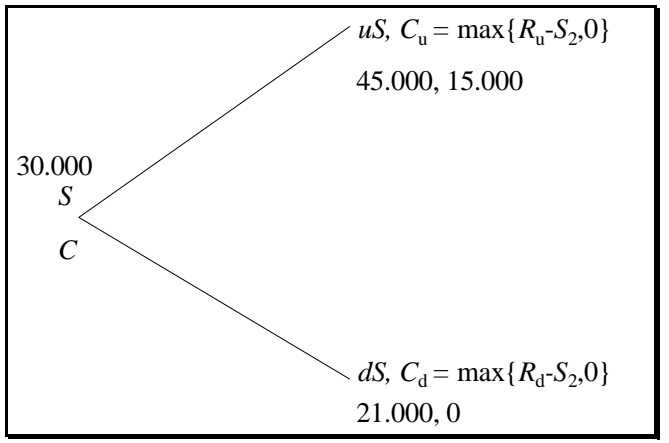

Abbildung 1: Entwicklung der Referenzanlage und des Optionspreises

Das Unternehmen wird die Option zum Zeitpunkt $t=1$ nur dann ausüben, wenn ein positiver Überschuß der Rückflüsse über den $\mathrm{zu}$ zahlenden Basispreis verbleibt. Dies ist nur im günstigen Umweltzustand der Fall. Es gilt ja $R_{u}-S_{2}>0$ und $R_{d}-S_{2}<0$. Es wurde bereits angedeutet, daß Arbitragefreiheit eine zentrale Annahme der bekannten Optionspreismodelle darstellt. Insofern ist nun zu prüfen, ob es eine handelbare Anlage gibt, deren erwartete Auszahlungsströme zum Zeitpunkt $t=1$ mit $R_{u}$ und $R_{d}$ übereinstimmen. Dies soll zunächst unterstellt werden. Wir nehmen an, daß eine derartige Anlage einen gegenwärtigen Wert von $S=30.000$ Geldeinheiten besitzt.

Nach diesen Annahmen ist die Bewertung der Option mit dem Binomialmodell von Cox et al. problemlos möglich (vgl. Cox et al. 1979). Je nach eintretendem Umweltzustand besitzt unsere Referenzanlage zum Zeitpunkt $t=1$ einen Wert von $u S=R_{u}$ oder $d S=R_{d}$. Daraus errechnet man die Größen $u$ und $d$, nämlich $u$ $=1,5$ und $d=0,7$. Abb. 1 zeigt die Entwicklung der Referenzanlage und die Entwicklung der zugehörigen Calloption.

Zur Bestimmung des Optionswertes $C$ konstruiert man ein Portfolio bestehend aus $M$ "Stück" der Referenzanlage und der risikofreien Anlage (risikofreier Zinssatz $r_{f}$ ) im Umfang von $B$. Es muß dann gelten:

$M u S+B\left(1+r_{f}\right)=C_{u}, M d S+B\left(1+r_{f}\right)=C_{d}$.

Dies sind zwei lineare Gleichungen in den zwei Unbekannten $M$ und $B$. Man erhält sofort

$$
M=\frac{C_{u}-C_{d}}{S(u-d)}, \quad B=\frac{u C_{d}-d C_{u}}{(u-d)\left(1+r_{f}\right)} .
$$

Positive Werte von $B$ bedeuten dabei Geldanlage, negative Werte entsprechend Geldaufnahme zum risikofreien Zinssatz $r_{f}$. Aufgrund der Annahme der Arbitragefreiheit besitzen Anlagen mit gleichem Auszahlungsspektrum auch den gleichen Wert. Demnach muß also $C=M S+B$ gelten. Daraus erhält man die Optionspreisformel des Binomialmodells: 


$$
C=\frac{1}{\left(1+r_{f}\right)}\left(\frac{1+r_{f}-d}{u-d} C_{u}+\frac{u-r_{f}-1}{u-d} C_{d}\right)
$$

Zur Bewertung der Option benötigt man folglich weder einen risikoangepaßten Diskontierungszinssatz noch die Schätzung beziehungsweise Kenntnis von Eintrittswahrscheinlichkeiten der zufällig verteilten Umweltzustände. Dies stellt einen wichtigen Vorteil dieser Methode dar. Andererseits ist die Kenntnis des Marktpreises der risikobehafteten Zahlungsströme $R_{u}$ und $R_{d}$ erforderlich. Die Bestimmung beziehungsweise Schätzung dieses Marktpreises kann in der Praxis problematisch sein.

Bevor dieses Problem näher diskutiert wird, soll zuvor noch $C$ für das gewählte Beispiel bestimmt werden. Unterstellt wird ein risikofreier Zinssatz $r_{f}=0.05$. Man erhält $M=0.625$ und $B=-12.500,00$. Daraus ergibt sich $C=6.250$. Da $C>$ $S_{1}=5.000$ gilt, ist es günstig, die Option zu erwerben. Die Differenz in Höhe von 1.250 Geldeinheiten entspricht dabei dem um Handlungsspielräume erweiterten Kapitalwert. Die Option sollte bei Eintreten des günstigen Umweltzustandes ausgeübt werden. Im anderen Fall verfällt die Option. Das präsentierte Beispiel zeigt, wie ein Pilotprojekt mit ex ante negativem Kapitalwert aufgrund der mit dem Projekt verbundenen Handlungsoptionen dennoch vorteilhaft ist.

Die Vorteilhaftigkeit wurde für $r<0.5$ auch mit dem Entscheidungsbaumverfahren nachgewiesen. Dies dürfte im Normalfall immer erfüllt sein. Allerdings genügen bereits kleine Veränderungen der Parameterwerte, um die obere Schranke für $r$ deutlich zu verringern. Setzt man etwa ceteris paribus $R_{u}=$ 41.000, so muß bereits $r<0,1$ für die Vorteilhaftigkeit gelten. Bei den Berechnungen im Rahmen des Entscheidungsbaumverfahrens wurde darüber hinaus die Eintrittswahrscheinlichkeit $p=0.5$ als bekannt vorausgesetzt. Diese Wahrscheinlichkeit wird im Rahmen der Optionspreistheorie nicht benötigt, an ihre Stelle tritt die Schätzung des Wertes einer marktfähigen Referenzanlage. Eintrittswahrscheinlichkeiten und Diskontierungszinssatz sind in der Größe $S$, also im Marktpreis implizit enthalten. Somit sind im Rahmen des Binomialmodells weniger Parameter zu schätzen. Dies erleichtert eventuelle Sensitivitätsanalysen beträchtlich.

Schließlich soll nochmals darauf hingewiesen werden, daß der bei Anwendung der Kapitalwertmethode erforderliche Diskontierungsfaktor von der risikobehafteten Zahlungsstruktur abhängig ist. Selbst wenn es dem Unternehmen gelingt, den korrekten Diskontierungszinssatz für das Projekt mit Wert $S$ und unsicheren gleich wahrscheinlichen Rückflüssen $R_{u}=u S$ beziehungsweise $R_{d}=$ $d S$ nach einer Periode zu ermitteln, so gilt dieser Diskontierungszinssatz nicht für den risikobehafteten Zahlungsstrom der Calloption auf das Projekt. Dies kann mit den Daten des Beispiels verdeutlicht werden. Aus der Gleichung

$$
S=\frac{1}{2}\left(R_{u}+R_{d}\right)(1+r)^{-1}=\frac{1}{2}(u S+d S)(1+r)^{-1} S
$$


errechnet man den korrekten Diskontierungszinssatz für das Beispiel. Es gilt

$$
r=\frac{1}{2}(u+d)-1 .
$$

Man erhält $r=0,10$. Für den risikobehafteten Zahlungsstrom der Option muß dagegen

$$
C=\frac{1}{2} C_{u}(1+r)^{-1}
$$

gelten. Daraus ergibt sich der korrekte Diskontierungszinssatz $r=0,20$. Dies verdeutlicht nochmals, wie schwierig die richtige Wahl eines derartigen Zinssatzes ist. Es genügt keinesfalls, einen derartigen Zinssatz "unternehmensweit" festzuschreiben.

Abschließend bleibt zu klären, wie man zu Schätzungen der Größe $S$ kommen kann. Diese Problematik wurde in der Literatur intensiv diskutiert. Gehr (vgl. Gehr 1981) zeigt, daß bei Vorliegen eines vollständigen Kapitalmarktes beliebige Zahlungsstrukturen aus vorhandenen Anlagemöglichkeiten kombiniert und damit bewertet werden können. Unterstellt wird dabei allerdings nach wie vor die Marktfähigkeit aller risikobehafteten Anlagemöglichkeiten. Empirische Untersuchungen zeigen, daß nicht marktfähige Anlagen bei gleichem nicht diversifizierbarem Risiko eine unterdurchschnittliche Rendite aufweisen (vgl. McDonald/Siegel 1984). Insofern kommt es zu einem Abschlag für mangelnde Marktgängigkeit beziehungsweise Liquidität. McDonald und Siegel (vgl. McDonald/Siegel 1984) zeigen, wie dieser Abschlag im Rahmen der Optionspreistheorie berücksichtigt werden kann. Die Modellierung des Abschlages erfolgt in der Form von (fiktiven) Dividendenzahlungen, für welche grundsätzlich die Halter von Optionen nicht entschädigt werden. Demnach liegt folgende Vorgehensweise nahe (vgl. auch die Ausführungen in Copeland et al. 1996, S. 464 ff. und Kilka 1995, S. 77 f.):

- Zunächst erfolgt unter der Annahme der Marktfähigkeit eine Schätzung von $S$. Dazu kann beispielsweise auf Branchendaten (eigenes Beta beziehungsweise Beta der Mitbewerber o.ä.) zurückgegriffen werden.

- Im zweiten Schritt wird aufgrund fehlender Marktgängigkeit ein Abschlag vorgenommen.

- Nach Berechnung des Optionspreises wird eine Sensitivitätsanalyse vorgenommen. Es wird unterstellt, daß die Größe $S$ innerhalb bestimmter Grenzen um einen Mittelwert schwankt. Auf Basis dieser Annahme wird mit Simulationstechniken die Verteilung des Optionspreises empirisch ermittelt. Dann können Aussagen über die Vorteilhaftigkeit des Projektes getroffen werden (vgl. Abschnitt 5).

Insgesamt ist festzuhalten, daß die Schätzung des Preises der Referenzanlage schwierig ist. Allerdings dürfte das vorgestellte Verfahren zur Schätzung insgesamt objektiver als die Schätzung von Eintrittswahrscheinlichkeiten und risikoangepaßten Diskontierungsfaktoren sein. Darüber hinaus sind nur die 
Parameter $S, u S$ und $d S$ zu schätzen, während im Falle der Anwendung des Entscheidungsbaumverfahrens $u S, d S$, Eintrittswahrscheinlichkeiten und der Diskontierungszinssatz zu bestimmen sind.

\section{Beurteilung von mehrstufigen Entwicklungs- projekten}

Es wird unterstellt, daß die Entwicklung eines IV-Systems auf der Basis eines Vorgehensmodells erfolgt. Im einfachsten Fall besteht ein derartiges Vorgehensmodell aus den vier Phasen Planung, Analyse, Design und Implementierung (vgl. dazu Stahlknecht/Hasenkamp 1997, S. 252 ff.). Am Ende einer jeden Phase besteht die Möglichkeit, das Projekt abzubrechen oder unter Zuführung weiterer Finanzmittel weiterzuführen. Diese Möglichkeit der Weiterführung kann als Calloption auf das Gesamtprojekt interpretiert werden. Insofern entsteht eine mehrstufige Folge aufeinander aufbauender Calloptionen (vgl. Abb. 2), sogenannter Compound-Optionen. Dabei hat die zweite (dritte) Option nur dann einen Wert, wenn die erste (erste und zweite) Option ausgeübt wird (werden). Insofern kann man diese Handlungsoptionen nicht separat bewerten und anschließend aufaddieren (vgl. auch Geske 1979). Die Bewertung der vorliegenden Compound-Option kann auf der Basis eines verallgemeinerten Binomialmodells erfolgen. Nachfolgend wird die einfachste mögliche Bewertungsmethode präsentiert. Mögliche Modifikationen beziehungsweise Erweiterungen werden im sechsten Abschnitt skizziert.

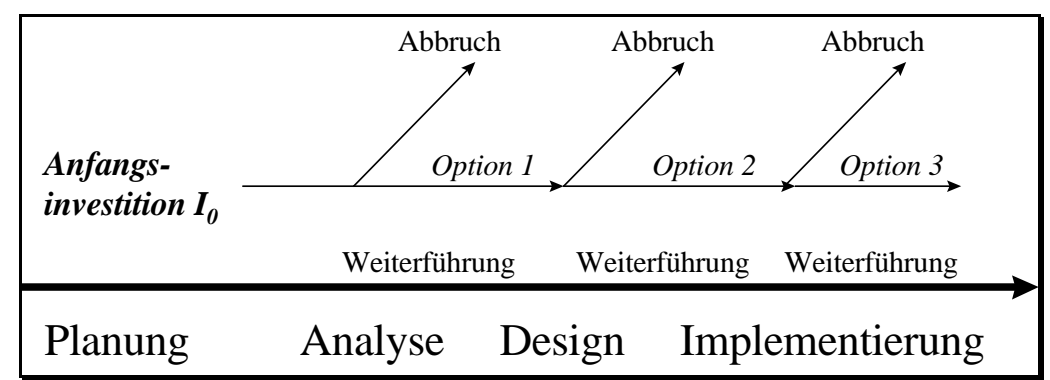

Abbildung 2: Folge von Weiterführungsoptionen im Rahmen eines Phasenmodells 


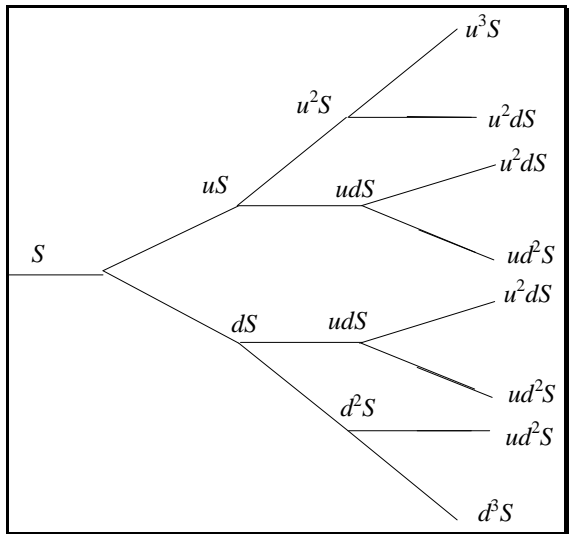

Abbildung 3: Unterstellte Entwicklung der Referenzanlage

Mit $I_{t}$ werden im folgenden die notwendigen Investitionen in Phase $t$ bezeichnet. Für $t=1,2,3$ ist $I_{t}$ dann auch der Basispreis der $t$-ten Weiterführungsoption. Der Einfachheit halber wird unterstellt, daß jede Phase exakt eine Zeitperiode dauert. Der risikofreie Zinssatz sei erneut $r_{f}$. Die Referenzanlage, welche den Wert der Rückflüsse des Entwicklungsprojektes exakt repliziert, besitzt in $t=0$ den Wert $S$. Sie folgt dem stochastischen Prozeß von Abb. 3. In jeder Periode kann ein günstiger Umweltzustand eintreten. Dann erhöht sich der Wert der Referenzanlage um den Faktor $u$. Andernfalls vermindert sich der Wert der Referenzanlage um einen Faktor $d$. Die Berechnung des Wertes der CompoundOption erfolgt rekursiv beginnend mit der letzten Periode $t=3$. Die dort auftretenden vier Optionen (vgl. Abb. 4) entsprechen der im dritten Abschnitt diskutierten Calloption und können völlig analog errechnet werden.

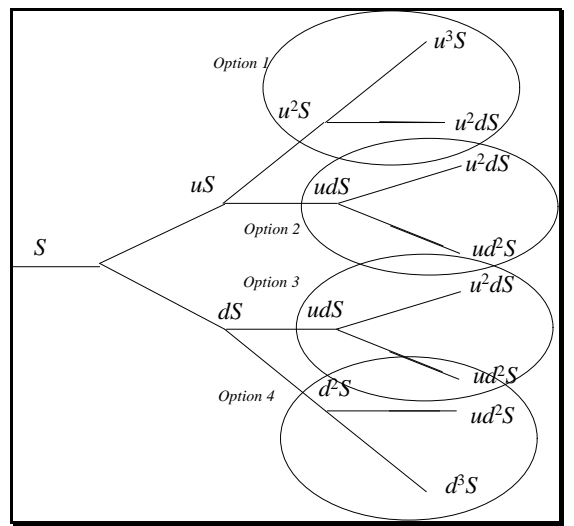

Abbildung 4: Rekursive Berechnung des Wertes der Compound-Option - erster Schritt 
Zur Berechnung der vierten Option in Abb. 4 bestimmt man beispielsweise zunächst die Größen

$$
C_{u, 4}^{3}=\max \left\{u d^{2} S-I_{3}, 0\right\}, C_{d, 4}^{3}=\max \left\{d^{3} S-I_{3}, 0\right\}
$$

und errechnet mit der im letzten Abschnitt hergeleiteten Optionspreisformel den Wert

$$
C_{4}^{2}=\frac{1}{\left(1+r_{f}\right)}\left(\frac{1+r_{f}-d}{u-d} C_{u, 4}^{3}+\frac{u-r_{f}-1}{u-d} C_{d, 4}^{3}\right)
$$

Der obere Index steht dabei für die Zeitperiode, der untere Index nummeriert die jeweilige Option. Analog errechnet man die Werte der verbleibenden drei Optionen.

Abb. 5 verdeutlicht den nächsten Schritt. Zur Berechnung der ersten Option zum Zeitpunkt $t=1$ errechnet man die Größen

$$
C_{u, 1}^{2}=\max \left\{C_{1}^{2}-I_{2}, 0\right\}, C_{d, 1}^{2}=\max \left\{C_{2}^{2}-I_{2}, 0\right\} \text {. }
$$

Eine erneute Anwendung des Binomialmodells liefert dann den Optionspreis $C_{1}^{1}$. Nachdem eine vollständige Ermittlung der Größen $C_{k}^{t}$ erfolgt ist, können die optimalen Weiterführungs- und Abbruchentscheidungen abgelesen werden. Dort wo ein Optionspreis den Wert 0 besitzt, ist abzubrechen. Die Anfangsinvestition $I_{0}$ ist nur zu leisten, wenn $C>I_{0}$ gilt.

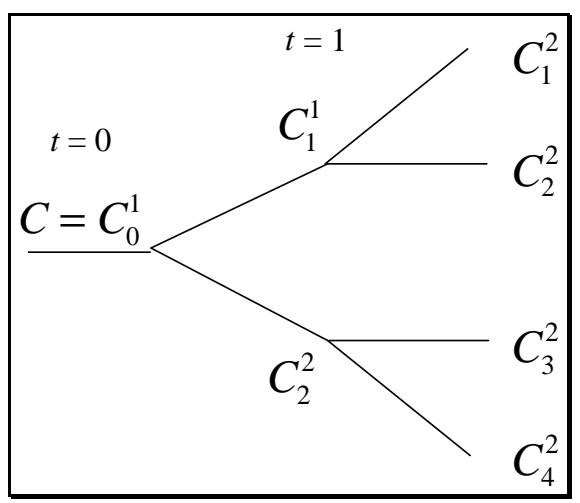

Abbildung 5: Rekursive Vorgehensweise zur Berechnung des Optionspreises

Zur Illustration der Vorgehensweise wird ein weiteres Beispiel betrachtet. Es gelte $I_{0}=1.000, I_{1}=10.000, I_{2}=8.000, I_{3}=5.000$. Das Unternehmen schätzt den heutigen Wert der Projektrückflüsse auf $S=20.000$. Bei günstiger Entwicklung während einer Entwicklungsperiode wächst der Wert Rückflüsse um den Faktor $u=1,2$, andernfalls fällt dieser Wert um den Faktor $d=0,7$. Der 
risikofreie Zinssatz betrage erneut $r_{f}=0,05$. Abb. 6 zeigt die errechneten Optionswerte auf Basis der angegebenen Daten. Demnach erfolgt ein Projektabbruch zum Zeitpunkt $t=1$, falls der negative Umweltzustand eintritt. Nach dieser Periode wird das Projekt in jedem Falle zu Ende geführt.

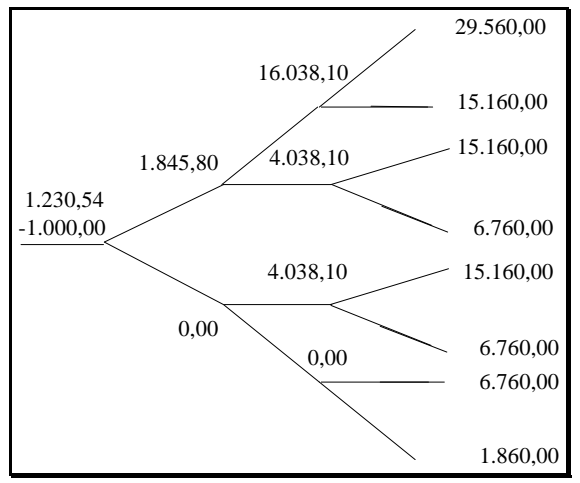

Abbildung 6: Ergebnisse für Beispiel

Was hätte eine Kalkulation des Entwicklungsprojektes ohne Berücksichtigung von Handlungsspielräumen ergeben? Schätzt das Unternehmen die Wahrscheinlichkeit für das Eintreten eines günstigen Umweltzustandes auf $p=$ 0,8 , so kann auf Basis dieser Schätzung der zugehörige Diskontierungszinssatz $r$ ermittelt werden. Es gilt nämlich die Beziehung

$$
S=S(1+r)^{-3} \sum_{k=0}^{3}\left(\begin{array}{l}
3 \\
k
\end{array}\right) p^{k}(1-p)^{3-k} u^{k} d^{3-k}
$$

Daraus ergibt sich $r=0,10$. Für den Kapitalwert erhält man demnach

$$
K=-\sum_{t=0}^{3} I_{t}(1+r)^{-t}+\sum_{k=0}^{3}\left(\begin{array}{l}
3 \\
k
\end{array}\right) p^{k}(1-p)^{3-k} u^{k} d^{3-k} S(1+r)^{-3} \text {. }
$$

Einsetzen der Werte liefert einen negativen Kapitalwert in Höhe von -459,03. Der Kapitalwert bleibt negativ für $r>0,082$. Unter Berücksichtigung der Handlungsoptionen beträgt der Projektwert nach Abzug der ersten Investitionsrate 230,54. Die Differenz in Höhe von 689,59 kann als Wert der mit dem Entwicklungsprojekt verbundenen Handlungsspielräume interpretiert werden. Das Beispiel zeigt nochmals, wie wichtig die Berücksichtigung von Handlungsspielräumen im Rahmen der Wirtschaftlichkeitsanalyse von Projekten ist. 


\section{Simulation}

Da eine genaue Schätzung der Parameter $S, u$ und $d$ im allgemeinen nicht möglich sein wird, sollte im Anschluß an die Berechnung des Optionswertes eine Sensitivitätsanalyse durchgeführt werden. Diese Analyse kann wie nachfolgend beschrieben ablaufen.

- Es wird eine Wahrscheinlichkeitsverteilung von $S$ postuliert. In vielen Anwendungen wird dabei $S$ als normalverteilt mit Erwartungswert $\mu$ und Standardabweichung $\sigma$ unterstellt. Diese Annahme erleichtert im allgemeinen die Generierung von Zufallszahlen. Allerdings ist $\mathrm{zu}$ beachten, daß bei Unterstellung dieser Annahme negative Werte von $S$ (gegebenenfalls mit sehr kleiner Wahrscheinlichkeit) möglich sind. Ebenso handelt es sich bei der Normalverteilung um eine symmetrische Verteilung, so daß die Modellierung eher pessimistischer beziehungsweise optimistischer Ausprägungen von $S$ (links- beziehungsweise rechtsschiefe Verteilungen) nicht möglich ist. Gegebenenfalls kann eine Beta-Verteilung unterstellt werden. Diese Verteilung hat positive Wahrscheinlichkeitsmasse innerhalb eines Intervalls [A,B], sie ist unimodal, erlaubt ansonsten aber die Modellierung nahezu beliebiger Verteilungsverläufe (insbesondere links- beziehungsweise rechtsschiefe Verteilungen unterschiedlicher "Stärke"). Dies wird in Abb. 7 für diverse Ausprägungen dieser Verteilung dokumentiert. Für eine detaillierte Behandlung sei auf die Literatur verwiesen (vgl. etwa Evans et al. 1993, S. $31 \mathrm{ff}$.).

- Nach Wahl der Verteilung werden Zufallszahlen generiert. Jede dieser Zufallszahlen beschreibt eine Ausprägung von $S$. Auf Basis dieses Wertes von $S$ wird dann der entsprechende Optionspreis ermittelt.

- Als Resultat erhält man einen Vektor von Optionspreisen zu verschiedenen Werten von $S$. Dadurch kann die Verteilung der Optionspreise angenähert werden. Schließlich können die üblichen Parameter wie Mittelwert, Varianz, Standardabweichung, Schiefe usw. des Optionspreises bestimmt werden. 


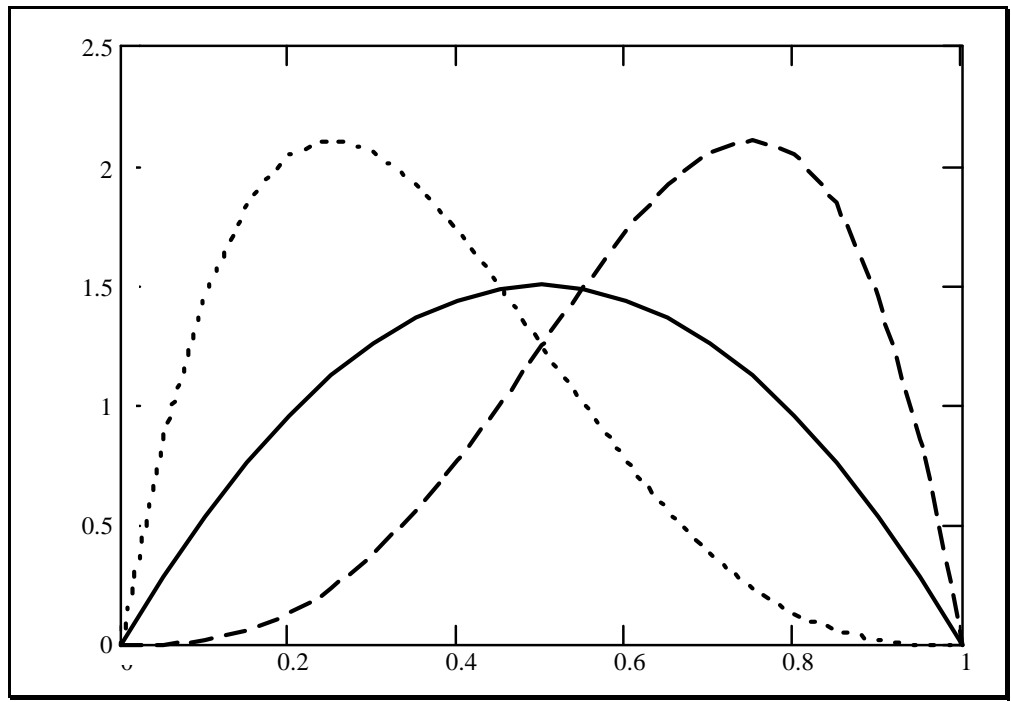

Abbildung 7: Diverse Ausprägungen der Beta-Verteilung (symmetrisch, links- beziehungsweise rechtsschief)

Eine formale Herleitung der Verteilung des Optionswertes scheitert im allgemeinen, da dieser keine lineare Funktion von $S$ darstellt. Charakteristisch für Optionen ist ja gerade die nichtlineare Abhängigkeit vom zugrunde liegenden Vermögensgegenstand. Realoptionen erlauben eine "Kappung" der Verluste bei negativen Umweltzuständen.

Nachfolgend werden für die Beispiele des dritten und vierten Abschnittes Sensitivitätsanalysen durchgeführt.

Im Beispiel des dritten Abschnittes unterstellen wir eine Normalverteilung von $S$ mit Mittelwert $\mu=30.000$ und Standardabweichung $\sigma=5.000$. Bekanntermaßen liegen dann die Werte von $S$ mit einer Wahrscheinlichkeit von 99\% in einem Intervall der Länge 5,14 $\sigma$ mit Mittelpunkt $\mu$. Im Beispiel liegen die Werte von $S$ damit nahezu sicher zwischen 17.150 und 42.850. Diese große Bandbreite soll der mit einem innovativen Pilotprojekt verbundenen Unsicherheit Rechnung tragen. Insgesamt wurden 2.000 Zufallszahlen generiert. Ein Histogramm der generierten Zufallszahlen zeigt Abb. 8. Der Mittelwert der Stichprobe betrug $m=$ 29.959,8, die Standardabweichung $s=4.910,8$. Die Daten der Stichprobe entsprechen damit nahezu exakt den Vorgaben.

Für jedes Element der Stichprobe wurde nun der zugehörige Optionswert ermittelt. (vgl. unteres Diagramm in Abb. 8). Da der Wert einer Calloption im ungünstigsten Fall 0 sein kann, ergibt sich dort eine Häufung. Der Mittelwert der Stichprobe beträgt $m=6.254,35$, die Standardabweichung $s=3011,0$. Das Projekt ist nur vorteilhaft, falls der Wert der Option über der Anfangsinvestition in Höhe von $S_{1}=5.000$ liegt. Die Wahrscheinlichkeit dafür beträgt $p=0,62$. 


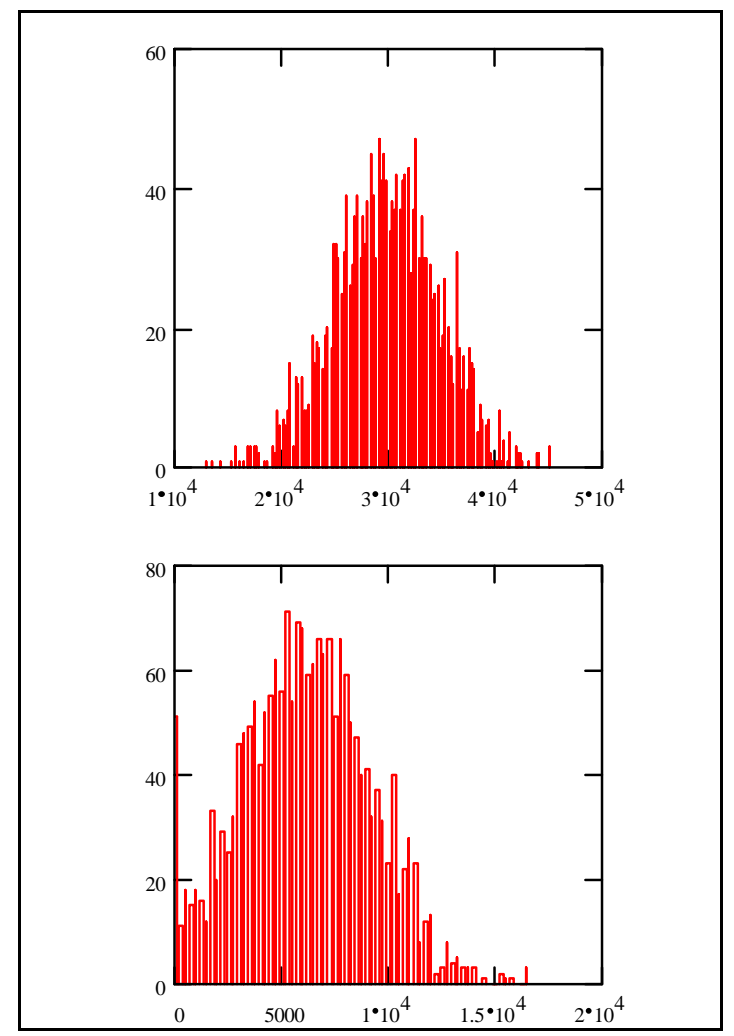

\section{Abbildung 8: Verteilung der Werte der simulierten Stichprobe (oben) und des Optionspreises (unten)}

Bei der Sensitivitätsanalyse für das Beispiel des vierten Abschnittes soll zur Illustration die Beta-Verteilung verwendet werden. Die Intervallgrenzen der Beta-Verteilung, die einem optimistischen und einem pessimistischen Wert entsprechen seien $A=10.000$ und $B=30.000$. Erneut werden 2.000 Zufallszahlen generiert. Dabei wurde zunächst eine symmetrische Beta-Verteilung unterstellt (genauer: $\beta(2,2)$ ist definiert auf $[0,1]$ und hat den Erwartungswert 0,5 und die Varianz 0,05; es erfolgte die Transformation $A+(B-A) \beta$ auf das Intervall [18.000,22.000]; daraus resultiert der Mittelwert 20.000 und die Standardabweichung 894,4; vgl. Evans et al. 1993, S. 31). Der Mittelwert entspricht dem im Beispiel verwendeten Wert von $S=20.000$. Abb. 9 zeigt die Verteilung der Stichprobe $S$ (oberes Diagramm) und der korrespondierenden Optionswerte (unteres Diagramm). Die Wahrscheinlichkeit, daß das Projekt durchgeführt werden sollte beträgt $p=0,59$.

Unterstellt man ein optimistischeres Szenario, so sollte man mehr Wahrscheinlichkeitsmasse auf größere Werte von $S$ legen. Man erhält dann eine 
rechtsschiefe Verteilung. Es gelte immer noch $A=18.000$ und $B=22.000$. Im Beispiel wurde die in Abb. 10 dargestellte obere Verteilung gewählt (dies entspricht einer Beta-Verteilung $\beta(3,2)$, die auf das Intervall $[A, B]$ transformiert wurde; der Mittelwert beträgt 20.400, die Standardabweichung 800; vgl. Evans et al. 1993, S. 31). Die Verteilung der Optionswerte ist im unteren Diagramm von Abb. 10 dargestellt. Die Wahrscheinlichkeit für einen positiven Projektwert ist natürlich höher als im vorher betrachteten Fall. Sie beträgt jetzt $p=0,75$.

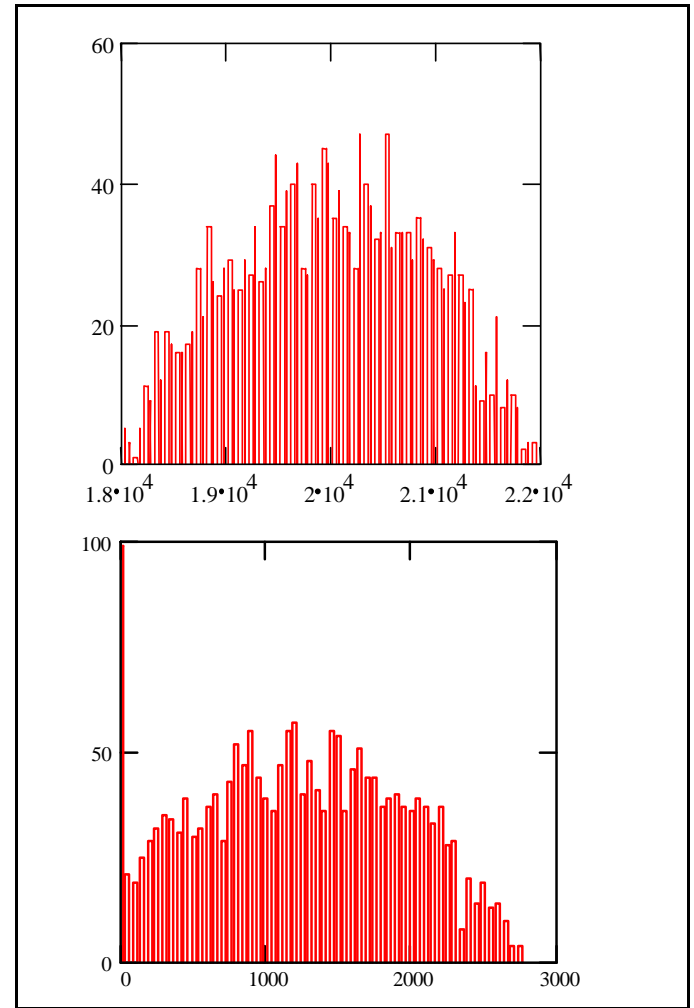

Abbildung 9: Verteilung des Preises der Referenzanlage (oben) und der korrespondierenden Optionspreise (unten) 


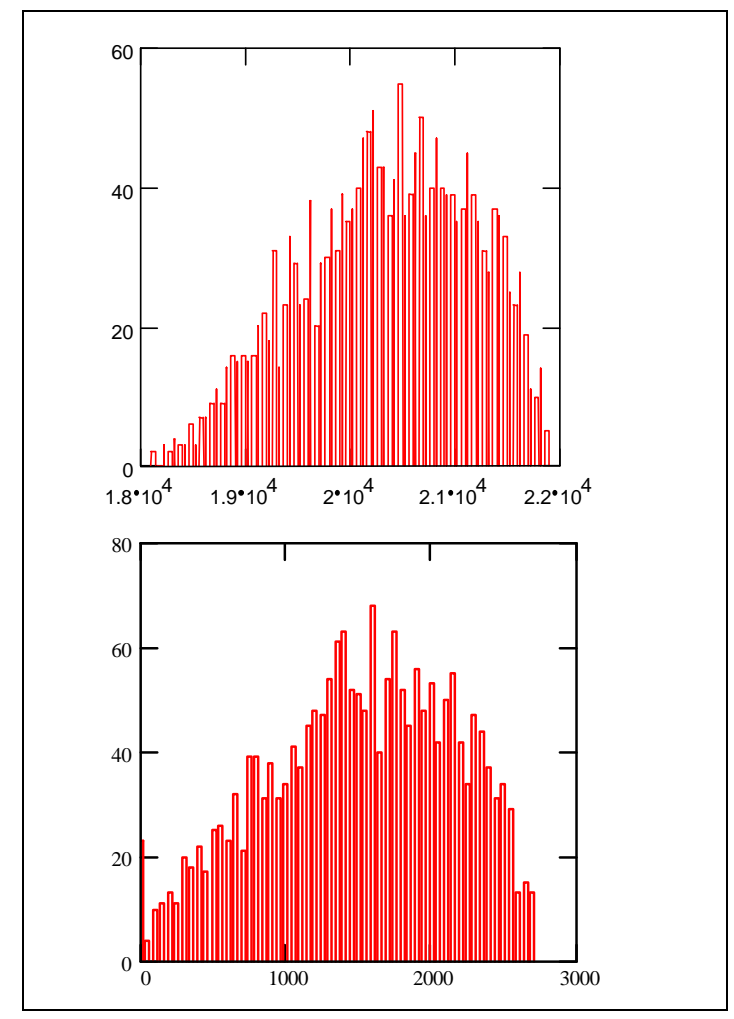

Abbildung 10: Verteilung des Preises der Referenzanlage (oben) und der
korrespondierenden Optionspreise (unten) in einem optimistischen Szenario

Die vorgestellten Beispiele zeigen exemplarisch, wie Simulationstechniken zur Sensitivitätsanalyse genutzt werden können. Um die Vorteilhaftigkeit eines Projektes abschätzen zu können, sind derartige Analysen unverzichtbar. Für den mit der Materie vertrauten Analysten stellen die handelsüblichen mathematischen Kalkulationsprogramme effiziente Werkzeuge dar (im vorliegenden Fall wurde das Produkt Mathcad der Firma MathSoft) verwendet. Für den weniger Geübten bedarf es einer entsprechenden Benutzerführung. In diesem Zusammenhang ist zu überlegen, das Instrumentarium der Realoptionen in der Form eines Entscheidungsunterstützungssystems zur Verfügung zu stellen. Dann können auch kompliziertere Optionskonstrukte bewertet werden. Dazu zählt beispielsweise die Option, Projektentscheidungen innerhalb eines bestimmten Zeitrahmens zu verzögern. Diese Option stellt eine sogenannte amerikanische Calloption dar, die jederzeit während der Laufzeit ausgeübt werden kann. Im Gegensatz dazu waren die hier betrachteten Optionen europäische Optionen. Eine Ausübung war nur am Tage der Fälligkeit möglich. Die Modellierung als europäische Calloptionen wurde aufgrund der guten Strukturierung von IV-Entwicklungsprojekten möglich. Bei der Untersuchung 
von Pilotprojekten wäre selbstverständlich auch die Berücksichtigung von Aufschuboptionen möglich. Im Gegensatz zu europäischen Optionen können bei amerikanischen Optionen Lösungen grundsätzlich nicht exakt in geschlossener Form berechnet werden. Im allgemeinen sind dazu numerische iterative Verfahren notwendig.

Die speziellen Eigenschaften der Beta-Verteilung erlauben die Modellierung und anschließende Simulation nahezu beliebiger Szenarien. Die Wahrscheinlichkeit, $\mathrm{da} ß$ das zugrunde liegende Projekt einen positiven Wert aufweist, kann aus der empirischen Verteilung leicht errechnet werden und stellt eine wichtige Information für die Entscheidungsträger des Unternehmen dar.

Neben $S$ können im Rahmen von Simulationsrechnungen auch die Parameter $u$ und $d$ variiert werden. In diesem Fall müssen allerdings Annahmen über die Korrelation der Größen $S, u$ und $d$ getroffen werden. Die Rechenzeiten für vergleichbare Simulationsläufe nehmen deutlich zu. Ebenso wird die Interpretation der Ergebnisse wesentlich schwieriger. Gegebenenfalls sollte in diesem Fall ein kontinuierliches Optionspreismodell verwendet werden (vgl. den folgenden Abschnitt).

\section{Ausblick}

Die den präsentierten Modellen zugrunde liegenden Annahmen können auf vielfältigere Weise modifiziert und dadurch realitätsnäher gestaltet werden.

- Einführung periodenspezifischer Größen $u$ und $d$. Im Falle von Entwicklungsprojekten erhält man so den in Abb. 11 dargestellten Pfad für die Referenzanlage mit dem Ausgangspreis $S$. Bei der Rückrechnung sind dann in der j-ten Periode die Größen $u_{j}$ beziehungsweise $d j$ zu anstelle von $u$ und $d \mathrm{zu}$ verwenden. Eine analoge Verallgemeinerung ist für den risikofreien Zinssatz rf möglich. Es muß allerdings kritisch hinterfragt werden, ob eine derartige Detaillierung wirkliche Vorteile bringt. Die Parameter $u$ und $d$ stellen (grobe) Schätzwerte dar. Es kann bezweifelt werden, ob eine sinnvolle Differenzierung dieser Parameter (ohne Willkür) möglich ist.

- Berücksichtigung unterschiedlicher Zeitdauern der einzelnen Phasen. Diese Erweiterung ist ebenfalls problemlos implementierbar. Dauert beispielsweise die letzte Phase zwei Perioden, so enthält der Pfad der Referenzanlage eine weitere Ebene (im Beispiel des IV-Entwicklungsprojektes eine vierte Ebene). Die Entscheidungen über die Fortführung im Rahmen der Analyse und Designphase fällt wie bisher zum Zeitpunkt $t=1$ beziehungsweise $t=2$. Die Entscheidung über die Implementierung könnte jetzt innerhalb des Zeitintervalls $[3,4]$ fallen. Da die letzte Investitionsrate allerdings erst zum Zeitpunkt $\mathrm{t}=4$ fällig ist, sollte auch erst dann über die Weiterführung entschieden werden. Eine frühere Festlegung wäre demnach nicht optimal. Zum Zeitpunkt $\mathrm{t}=3$ besteht deshalb nur formal eine Weiterführungsbeziehungsweise Abbruchoption mit Basispreis 0. Anzumerken ist, daß der 
Rechenaufwand stark ansteigt (jede zusätzliche Periode bedeutet im Prinzip eine Verdoppelung der Größe des Baumes in Abb. 3 beziehungsweise Abb. 12). Gegebenenfalls sollte in derartigen Fällen wiederum ein kontinuierliches Modell der Optionspreisbewertung zugrunde gelegt werden.

- Berücksichtigung von Aufschuboptionen. Bei zahlreichen Entwicklungsprojekten kann die Entscheidung über die Fortführung eines Projektes zeitlich aufgeschoben werden. In diesem Falle wird eine Entscheidung nicht zu einem vordefinierten Zeitpunkt $\mathrm{t}=\mathrm{t} 0$ getroffen, sondern innerhalb eines Zeitintervalls $[\mathrm{t}, \mathrm{t}+\mathrm{h}]$. Es liegt eine amerikanische Option vor. Geschlossene Lösungen können im allgemeinen nicht mehr errechnet werden. Vielmehr sind geeignete numerische Verfahren notwendig. Sensitivitätsanalysen gestalten sich in solchen Fällen schwieriger und aufwendiger. Es ist in jedem Fall günstiger, ein kontinuierliches Modell zu verwenden (vgl. Wilmott et al. 1995, S. 71 ff. und S. 106 ff.).

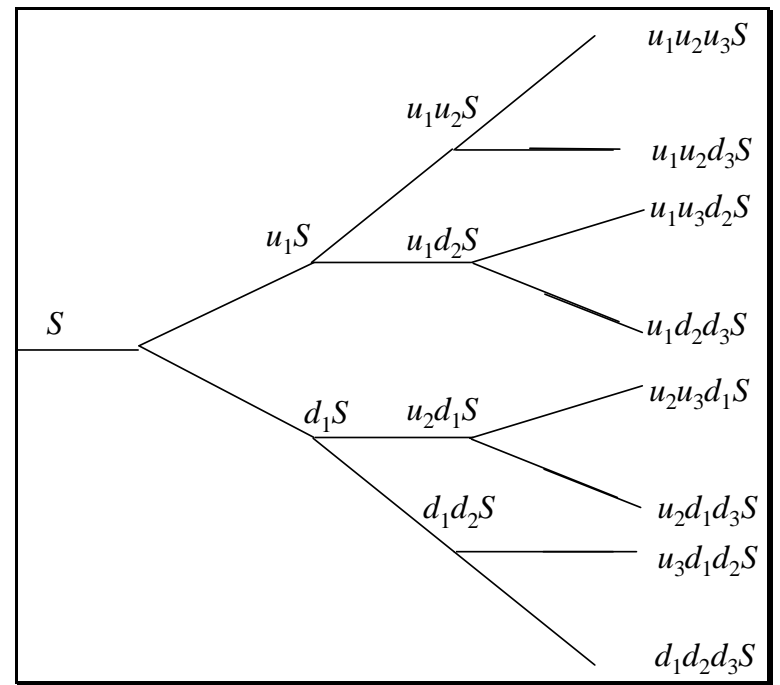

\section{Abbildung 11: Berücksichtigung periodenspezifischer Parameter $u_{j}$ und $d_{j}$}

Bereits mehrfach wurde die Möglichkeit der Verwendung eines kontinuierlichen Optionspreismodells als Alternative erwähnt. Dabei postuliert man im allgemeinen, daß die Kursentwicklung der Referenzanlage einem Random Walk folgt. Genauer unterstellt man die Beziehung

$$
d S=\mu S d t+\sigma S d X
$$

(vgl. Wilmott et al 1995, S. 20 ff.). Die Größe $\mu$ stellt den (im allgemeinen positiven) Trend (dieser entspricht der erwarteten Rendite) und $\sigma$ die Volatilität der Referenzanlage dar. $X$ ist ein Standard-Wiener-Prozeß und repräsentiert einen stochastischen Störterm (vgl. Dixit/Pindyck 1993, S. 59 ff. für eine anschauliche Darstellung). Auf Basis der unterstellten Preisentwicklung der Referenzanlage 
kann der Preis einer europäischen Option abgeleitet werden. Man erhält so die bekannte Black-Scholes-Formel (vgl. Copeland/Weston 1992, S. 267-269). Die Darstellung der Referenzanlage in der skizzierten Form erlaubt einfache numerische Simulationen. Die Ergebnisse sind dann auch leichter auf den Fall der Bewertung eines amerikanischen Calls auf die Referenzanlage übertragbar (vgl. Wilmott et al. 1993, S. 106 ff.).

Der kontinuierliche Bewertungsfall kann aus dem in der vorliegenden Arbeit skizzierten diskreten Fall durch Grenzübergang erhalten werden. Dazu werden bei konstantem Zeithorizont $T$ die Periodendauern sukzessive halbiert. Dixit und Pindyck (vgl. Dixit/Pindyck 1993), S. 68 ff., demonstrieren die Herleitung und den "Übergang" der Parameter $u$ und $d$ auf $\mu$ und $\sigma$. Die Verwendung kontinuierlicher Modelle erleichtert Simulationsrechnungen und senkt den notwendigen Rechenaufwand. Andererseits ist die zugrunde liegende Theorie stochastischer Prozesse schwierig zugänglich und kaum anschaulich vermittelbar. Dies stellt ein gravierendes Defizit für die Akzeptanz in der Praxis dar und unterstreicht nochmals die Notwendigkeit der Entwicklung eines Entscheidungsunterstützungssystem, welches dem Analysten theoretische Bausteine unter einer geeigneten Benutzerführung zur Verfügung stellt.

Der skizzierte Ansatz kann zur Bewertung von Entwicklungsprojekten für wettbewerbsorientierte IV-Systeme verwendet werden. In diesem Falle sind allerdings Aktionen potentieller Konkurrenten zu berücksichtigen, so daß spieltheoretische Methoden anzuwenden sind. Eine Einbindung der Optionspreistechniken in den VAR-Ansatz von Liang und Tang (vgl. Liang/Tang 1992), der speziell zur Bewertung wettbewerbsorientierter Systeme konzipiert wurde, wird gegenwärtig vorgenommen.

\section{Zusammenfassung}

Im vorliegenden Beitrag wurde gezeigt, wie die Anwendung der Optionspreistheorie $\mathrm{zu}$ einer realistischeren Bewertung von IV-Pilotbeziehungsweise IV-Entwicklungsprojekten führt. Die besonders gute Strukturierung von IV-Entwicklungsprojekten in Verbindung mit klar definierten Meilensteinen, an denen über die Weiterführung beziehungsweise den Abbruch eines Projektes entschieden werden kann, erleichtert die Berechnung des Wertes von Handlungsoptionen. Insofern ist der IV-Bereich als Anwendungsgebiet der Theorie der Realoptionen geradezu prädestiniert. Der vorgestellte Ansatz erlaubt die Bewertung von Handlungsspielräumen innerhalb von IV-Projekten auf Basis eines unterstellten Wertes der zukünftigen Rückflüsse (Referenzanlage). Der unterstellte Wert der Referenzanlage kann als erwarteter Kapitalwert zukünftiger Rückflüsse interpretiert werden. Die damit verbundene Schätzproblematik wurde diskutiert. Es wichtig, die ermittelten Ergebnisse im Rahmen einer Sensitivitätsanalyse auf ihre Stabilität zu untersuchen. Sensitivitätsanalysen 
bedürfen des Einsatzes von Simulationstechniken. Selbst bei Zugrundelegung einer "einfachen" Verteilung der Werte der Referenzanlage (etwa einer Normalverteilung) kann die Verteilung des Optionswertes aufgrund des nichtlinearen Charakters von Optionsrechten nicht mehr explizit hergeleitet werden. Zur Berechnung der Optionswerte und zur Durchführung der Simulationen können kommerziell verfügbare mathematische Spreadsheets benutzt werden. Diese setzen im allgemeinen Spezialkenntnisse der verwendeten mathematischen Verfahren voraus. Eine Verwendung des skizzierten Ansatzes in der Praxis erfordert demzufolge die Konstruktion eines Entscheidungsunterstützungssystems mit einer für mathematische Laien verständlichen Benutzerführung. An der Entwicklung eines derartigen Werkzeuges wird gegenwärtig gearbeitet. Das Werkzeug erlaubt die Bewertung der im Beitrag skizzierten Abbruchoptionen und darüber hinaus die Analyse von Optionen, ein Projekt zeitlich aufzuschieben (Aufschuboption).

Mathcad-Spreadsheets (Mathcad Plus Version 6.0) der skizzierten Ansätze sind beim Autor erhältlich. Die entwickelte Methodik wird gegenwärtig im Rahmen eines größeren Praxisprojektes validiert und im Hinblick auf ihre Praktikabilität untersucht.

\section{Literaturverzeichnis}

Antweiler, J.(1995): Wirtschaftlichkeitsanalyse von Informations- und Kommunikationssystemen auf der Basis von Wirtschaftlichkeitsprofilen. Information Management, 10(1995)4, S. 56-64.

Bamberg, G./ Coenenberg, A. (1996): Betriebswirtschaftliche Entscheidungslehre. 9. Aufl., München 1996.

Copeland, T./ Koller, T., Murrin, J. (1996): Valuation. Measuring and Managing the Value of Companies. New York 1996.

Copeland, T./ Weston, F. (1992): Financial Theory and Corporate Policy. $3^{\text {rd }}$ Ed., Reading, MA. 1992.

Cox, J./ Ross, S./ Rubinstein, M. (1997): Option Pricing: A Simplified Approach. J. of Financial Economics, 7(1979), S. 229-263.

Dixit, A./ Pindyck, R. (1993): Investment under Uncertainty. Princeton, NJ. 1993.

Evans, M./ Hastings, N./ Peacock, B. (1993): Statistical Distributions. $2^{\text {nd }}$ Ed., New York 1993.

Gehr, A. (1981): Risk-Adjusted Capital Budgeting Using Arbitrage. Financial Management, Winter 1981, S. 14-19.

Geske, R. (1995): The Valuation of Compound Options. Journal of Financial Economics, 7(1995), S. 32-54. 
Hull, J. (1998): Introduction to Futures and Options Markets. New York 1998.

Kambil, A./ Henderson, J./ Mohsenzadeh, H. (1995): Strategic Management of Information Technology Investments: An Option Perspective. In: Managing Information Technology Investments with Outsourcing. Ed.: M. Khsrowpour. Harrisburg, PA. 1995, S. 32-54.

Kilka, M. (1995): Realoptionen. Optionspreistheoretische Ansätze bei Investitionsentscheidungen unter Unsicherheit. Frankfurt am Main 1995.

Laux, C. (1993): Handlungsspielräume im Leistungsbereich des Unternehmens: Eine Anwendung der Optionspreistheorie. zfbf, 45(1993)11, S. 933-957.

Liang, T.-P./ Tang, M.-J. (1992): VAR-Analysis: A Framework for Justifying Strategic Information Systems Projects. DATABASE, 23(1992)1, S. 2735 .

McDonald, R./ Siegel, D. (1984): Option Pricing When the Underlying Asset Earns a Below-Equilibrium Rate of Return: A Note. Journal of Finance, 24(1984)1, S. 261-265.

Schumann, M. (1993): Wirtschaftlichkeitsbeurteilung für IV-Systeme. Wirtschaftsinformatik, 35(1993) 2, S. 167-178.

Spremann, K. (1996): Wirtschaft, Investition und Finanzierung. 5. Aufl., München, Wien 1996.

Stahlknecht, P./ Hasenkamp, U. (1996): Einführung in die Wirtschaftsinformatik. 9. Auflage. Berlin, Heidelberg 1996.

Stickel, E. (1992): Eine Erweiterung des hedonistischen Verfahrens zur Ermittlung der Wirtschaftlichkeit des Einsatzes von Informationstechnik. ZfB, 62(1992)7, S. 47-64.

Wilmott, P./ Howison, S./ Dewynne, J. (1995): The Mathematics of Financial Derivatives. Cambridge 1995.

Zangemeister, C. (1976): Nutzwertanalyse in der Systemtechnik. München 1976. 